\title{
Advancing Epidemic Prediction and Forecasting: A New US Government Initiative
}

\author{
Jean-Paul Chretien*1, David Swedlow ${ }^{2}$, Irene Eckstrand ${ }^{3}$, Dylan George ${ }^{4}$, Michael \\ Johansson ${ }^{2}$, Robert Huffman ${ }^{5}$ and Andrew Hebbeler ${ }^{6}$
}

${ }^{1}$ Armed Forces Health Surveillance Center, Silver Spring, MD, USA; ²Centers for Disease Control and Prevention, Atlanta, GA, USA; ${ }^{3}$ National Institutes of Health, Bethesda, MD, USA; ' ${ }^{B}$ iomedical Advanced Research and Development Authority, Washington, DC, USA; ${ }^{5}$ Department of Defense, Ft. Belvoir, VA, USA; ${ }^{6}$ Office of Science and Technology Policy, Washington, DC, USA

\section{Objective}

To accelerate the development of US federal infectious disease outbreak prediction (i.e., identification of future time and place of a disease event) and forecasting (i.e., disease spread) capabilities.

\section{Introduction}

The National Science and Technology Council, within the Executive Office of the President, established the Pandemic Prediction and Forecasting Science and Technology (PPFST) Working Group in 2013. The PPFST Working Group supports the US Predict the Next Pandemic Initiative, and serves as a forum to accelerate the development of federal infectious disease outbreak prediction and forecasting capabilities. Priorities include identification, evaluation, and integration of disparate biosurveillance and other data streams for prediction/forecasting; characterization of the decision context for US Government use of prediction/forecasting models; and development of a common US Government vision for federal prediction/forecasting capabilities. The Working Group comprises 18 federal departments and agencies, as well as the National Security Council, Office of Science and Technology Policy (OSTP), and Office of Management and Budget. OSTP, the Centers for Disease Control and Prevention, and the Department of Defense chair the Working Group.

\section{Methods}

In 2014, the Working Group initiated a pilot project, "Integrating Prediction and Forecasting Models for Decision-Making: Dengue Epidemic Prediction", to demonstrate the practical application of prediction and forecasting models. The first stage of the pilot project was a White House workshop in September 2014 including PPFST Working Group members and leading US and international experts in dengue surveillance, public health decision-making, and modelling. The goal of the workshop was to initiate a dengue prediction and forecasting effort using existing dengue surveillance and predictor data. A second workshop, in 2015, will evaluate model performance against datasets assembled and provided to modeling teams following the first workshop.

\section{Results}

Workshop participants considered public health and national security objectives for dengue epidemiological modelling, promising dengue models, and existing dengue surveillance datasets and other data (e.g., climatological) that could inform models. They developed a plan for evaluating the performance of the various models that will be applied to the data.

\section{Conclusions}

Through the initial dengue workshop, the PPFST Working Group identified data and models for the dengue pilot project, as well as technical and organizational needs for dengue prediction/forecasting. The Working Group believes that much of this knowledge also could be applied to future prediction/forecasting efforts for other pathogens.
PPFST Working Group leaders will present workshop outcomes, and seek feedback on the Working Group's direction from the ISDS community.

\section{Keywords}

Prediction; Forecasting; Federal

\section{Acknowledgments}

The views expressed are those of the authors and do not necessarily represent the views of any part of the US Government.

\author{
*Jean-Paul Chretien \\ E-mail: jpchretien@gmail.com
}

